\title{
Reduction of Reinfection Rates with Opisthorchis viverrini through a Three-Year Management Program in Endemic Areas of Northeastern Thailand
}

\author{
Suksanti Prakobwong ${ }^{1,2, *} \mathbb{E}$, Kulwadee Suwannatrai ${ }^{3}$ [D \\ 'Department of Biology, ${ }^{2}$ Northeast Liver Fluke Research and Outreach Unit, Faculty of Science, Udon Thani Rajabhat University, Udon Thani 41000, \\ Thailand; ${ }^{3}$ Department of Biology, Faculty of Science, Sakon Nakhon Rajabhat University, Sakon Nakhon 47000, Thailand
}

\begin{abstract}
To clarify the reinfection profile associated with risk factors of opisthorchiasis, we conducted an epidemiological study on the chemotherapeutic effects on reinfection with 0 . viverrini in the endemic areas of Northeastern Thailand for 3 years. A total of 3,674 fecal samples were collected from participants in villages of 5 provinces. They were examined microscopically using a modified technique of formalin ethyl-acetate concentration. Egg-positive residents were reexamined year (2018) by year (2019) after treatment with a single dose $(40 \mathrm{mg} / \mathrm{kg})$ of praziquantel. Health education was provided to the participants yearly. The egg-positive rate of $O$. viverrini was $14.3 \%$, and was highest (22.2\%) in the 20 -30 year-old group in 2017 . The egg positive rate was $15.3 \%$ in dogs and $11.4 \%$ cats. Human reinfection rate was $15.5 \%$ and $6.3 \%$ in next 2 years, and was highest (23.2\%) among the fishermen. Relative risk factors of reinfection were significantly higher for males, over 40-year-old age, or working as fishermen or farmers, and eating uncooked fish within the preceding year. A significant difference resulting from a health education program was observed in the third year. Therefore, health education and sustainable surveillance for opisthorchiasis should be maintained to decrease the risk of reinfection.
\end{abstract}

Key words: Opisthorchiasis, re-infection, reduction rate, chemotherapy, risk factor

\section{INTRODUCTION}

Reinfection of Opisthorchis viverrini promotes hepatobiliary diseases, especially cholangiocarcinoma. This is a critical problem that is endemic in Northeastern Thailand [1]. Several previous studies have found that patients repeated praziquantel administration and this is linked to the number of reinfections by O. viverrini, and increases the risk of cholangiocarcinoma [2,3]. People who took praziquantel showed an average risk 2.30 times higher than those who had never taken this drug [4]. The endemic region was identified as areas near water bodies and presented increased risks of reinfection [5-7]. There are large number of water bodies widely spread over the region which support communities $[5,6]$. In the past, prevention and control of opisthorchiasis were performed over short periods and discontinuously depending on the occurrence of outbreaks

- Received 6 April 2020, revised 22 September 2020, accepted 25 September 2020. *Corresponding author (sprakobwong@yahoo.com) (c) 2020, Korean Society for Parasitology and Tropical Medicine This is an Open Access article distributed under the terms of the Creative Commons Attribution Non-Commercial License (https://creativecommons.org/licenses/by-nc/4.0) which permits unrestricted non-commercial use, distribution, and reproduction in any medium, provided the original work is properly cited. and national policy. Although, the prevalence of opisthorchiasis in this region was diminished compared to previous times, the persistence of cholangiocarcinoma cases is still high in the region [1]. Therefore, prevention and control in endemic areas need to be done intensively and continuously to reduce repetitive infections with $O$. viverrini and associated cholangiocarcinoma.

The prospective risk factor associated with $O$. viverrini infection is consumption of raw or partially cooked fish in many kinds of traditional dishes. Evidence shows the distribution of the infective form, the metacercria, in the cyprinid fish of which infection rates were $7.7-70.0 \%$ in the Northeast region $[5,8,9]$. This alimentary behavior is influenced by indirect factors such as socioeconomic considerations, lifestyle, as well as the health education of the citizenry in Northeastern Thailand [10]. In endemic communities, it has been reported that reinfection with O. viverrini occurred after chemotherapies $[11,12]$. However, the reinfection profile in a long-term study with health education and indirect risk factors are not clear.

In northeastern provinces, the prevalence of $O$. viverrini reported during last decade decreased by 10-19\% [8]. The average incidence of chaolangiocarcinoma was 38 persons per 100,000 
in the Northeast [13]. However, complete epidemiological data for existing reservoir hosts has never been simultaneously determined in each of the endemic areas [5]. Additionally, a lack of reinfection data and recent information about the effect of health education in a longitudinal study needs to be further developed. The increased risk of opisthorchiasis-associated cholangiocarcinoma still remains unknown as a result of imprecise prevention and control strategies. The current work is a longitudinal study aimed to investigate 3 years of $O$. viverrini reinfections, integrating the prevalence and intensity of this illness in residents and the reservoir hosts. This included domestic dogs and cats, in and around certain water basins in the upper part of Northeastern Thailand. Fecal samples were collected in 54 villages. The number of eggs per gram of feces was assessed using a modified formalin ethyl-acetate concentration technique. Relative risk factors of reinfection were analyzed. Health education was provided to the participants yearly.

\section{MATERIALS AND METHODS}

\section{Ethics statement}

The protocol of the study was approved by the Human Ethics Committee of Udon Thani Rajabhat University (HECUD. 03/2017). Letters explaining the aims of programs and strategies were sent to village heads, community leaders, and individual households. A visitation to each village was made to explain the benefits of the survey and the study as a part of the national control program. The Animal Ethics Committee of Udon Thani Rajabhat University approved this part of the study (AREC. UDRU.01/2016).

\section{Study area and fecal sample collection}

This study was conducted in Thai villages over 3 years from April 2017 to December 2019 around the water resources in the upper part of Northeastern Thailand. These villages were located near the Mekong River (Nong Khai Province), Kud-thing Ramsar (Bueng Kan Province), Nam Oon Reservoir (Sakon Nakhon Province), Ubon Rattana Dam (Nong Bua Lam Phu Province), Nong Han Wetlands and Lam Pao River (Udon Thani Province). An epidemiological study of opisthorchiasis in humans was conducted as a primary outreach for sustainable prevention and as a control strategy by the Faculty of Science, Udon Thani Rajabhat University.

To evaluate the prevalence of liver fluke infections in the first year (April 2017), fecal samples were collected from 3,674 par- ticipants (1,244 males, 2,430 females) in 54 villages (age range, 20-76 years old). Participants were evaluated for inclusion in the study using questionnaires. Residents who ate raw fish, were not diagnosed with a parasitic infection within last 5 years and had an asymptotic disorder were included in this study. A visitation to each village was made to explain the method to collect fecal samples.

\section{Modified formalin ethyl-acetate concentration technique}

A quantitative modified formalin ethyl-acetated concentration technique was performed with slight modifications that involved increasing the volume of ethyl acetate used in the procedure. It also reduced the amount of $10 \%$ formalin to preserve the sediments and used of a higher sensitivity of detection for parasite eggs. Briefly, for each test, 0.5 gram of feces were fixed in $10 \mathrm{ml}$ of $10 \%$ formalin for 3 days and filtered through 2 layers of gauze. The suspension was centrifuged at 2,500 rpm for $3 \mathrm{~min}$. The resulting pellet was resuspended in $10 \mathrm{ml}$ of $10 \%$ formalin and $4 \mathrm{ml}$ of ethyl-acetate and then centrifuged at 2,500 rpm for $3 \mathrm{~min}$. A lipid layer floating in the solution was removed by tearing inside the centrifuge tube. The pellet was resuspended in $5 \mathrm{ml}$ of $10 \%$ formalin and centrifuged at $5,000 \mathrm{rpm}$ for $1 \mathrm{~min}$. The resulting sediment was added to 0.5 $\mathrm{ml}$ of $10 \%$ formalin. The number of liver fluke eggs per gram of feces was assessed and confirmed by 2 experienced researchers.

\section{Treatment and health education}

Human opisthorchiasis and all other parasitic infections among the participants were treated by effective parasitic drugs within 3 weeks after the sample collection by a physician with expertise in this area. The O. viverrini-infected residents were treated with single dose of praziquantel, $40 \mathrm{mg} / \mathrm{kg}$ body weight. After treatment in each year of the survey, fecal samples were randomly collected from $5 \%$ of the participants to investigate effect of this chemotherapy in one month after treatment. The questionnaire was used to collect demographic data. A KAP (knowledge, attitude and practices) survey was done that served as a base line assessment of individual participants to gauge the effectiveness of health education.

The researchers submitted a letter outlining the study and contacted the Health Promoting Hospital for permission to collect data and samples, and provide health education. In the first year, health education was provided to the $O$. viverrini-infected participants to encourage behavioral changes by increasing their knowledge and changing their attitudes. Information 
about parasitic infections, especially concerning liver fluke prevention and control, the severity of opisthorchiasis, liver tissue resorption after treatment, and the severity of cholangiocarcinoma were emphasized in health education sessions. In the second year, the Visit Home and We Eat Cooked Fish programs were done to change their behavior. In the third year, the Visit Home and Do Not Eat Raw Fish, No Risk to Disease programs were implemented. Health education was evaluated by pre-and post-test comparison on a yearly basis.

\section{Evaluation on reinfection and risk factors}

All O. viverrini-infected participants in the first year were included in a repeat infection investigation. Their participation was voluntary and done under human ethics informed consent. The fecal sample collection method was explained to the participants by village health volunteers. Reinfection data were evaluated yearly by diagnosis over the 3-year period, 2017, $2018(n=419)$

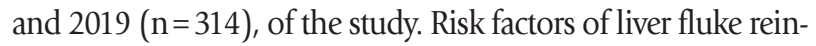
fection were evaluated in the second year (2018) using questionnaires inquiring about the gender, age, occupation, education, information about parasitic treatment, and eating behaviors of the participants $(\mathrm{n}=340)$. The new incidence assessment of infection in the second year from uninfected citizens was done randomly among the residents who lived near water bodies, collecting 50 samples in each province $(n=250)$.

\section{Investigation of $O$. viverrini infection in reservoir hosts}

Distribution of the liver flukes in the reservoir hosts is a key to maintain the parasitic cycle. The dogs and cats in villages close to water bodies was purposively selected. The owners of these animal reservoirs consented to sample collection. Fecal samples were collected from domestic dogs (247) and cats (61) in August 2017. The animals were caged in the homes of their owners in 21 villages. The stools of dogs and cats were collected after confining them, thus allowing the fecal sample results to be correlated with the data obtained from the human occupants of the same household. Gastrointestinal parasitic infections in the dogs and cats were examined using the modified formalin ethyl-acetate concentration technique. The O. viverrini infections were treated using a single dose of praziquantel at a level of 50 $\mathrm{mg} / \mathrm{kg}$ body weight. Other parasitic nematode infections were treated with $200 \mathrm{mg} / \mathrm{kg}$ of albendazole.

\section{Statistical analyses}

Data are presented as an average of percent and intensity of infection. The reinfection rate was examined in fecal samples of people infected in the first year of the study. One-way ANOVA was used to compare the intensity of the number of eggs per gram of feces (EPG). Student's t-test was used for parametric comparison. Pearson's correlation coefficient was employed to analyze correlations of parametric data. Regression analysis was done when the data had significant relationships. Odds ratios (OR) were calculated to determine the association between cross-sectional data to predict possible risk factors. The ORs were adjusted for all other predictive variables based on logistic regression. Statistical analyses were performed using SPSS Version 22. P-values less than 0.05 were considered statistically significant.

The administrative boundaries of Thailand and Udon Thani Province were downloaded from www.diva-gis.org. They were used as a base layer for creating opisthorchiasis prevalence and reinfection maps through ArcGIS mapping software (ESRI, Redlands, California, USA).

\section{RESULTS}

\section{Prevalence of opisthorchiasis in residents}

The study was performed under the development of a sustainable prevention and control strategy in the community by the Faculty of Science of Udon Thani Rajabhat University. The prevalence of O. viverrini infection was 14.3\% (EPG 83.7, range 25-2,274) among the villagers (Table 1). The highest rate was $34.6 \%$. The prevalence in males (15.4\%) was higher than in females (13.7\%). However, the overall intensity was significantly different in males (EPG 144.0) compared to in females (EPG 49.0). Our study found the highest infection rate in the 20-30 years old age group and the average intensity peaked in the age range of 41-50 years old (EPG 105.7, range $25-2,274)$. The majority positive cases were detected in Sakon Nakhon Province (16.0\%, EPG 125.5, range 68-2,274), and among fishermen (25.7\%, EPG 169.4, range 49-2,274). A spatial distribution of human $O$. viverrini infection in the first year (2017) is shown in Fig. 1.

\section{Reinfection status by surveyed year and areas}

The reinfection of human opisthorchiasis patients was evaluated in the second $(\mathrm{n}=419)$ and third years $(\mathrm{n}=314)$ as shown in Table 2. In the second year, after praziquantel treatment and health education, prevalence of reinfection was 15.5\% (EPG 21.4, range 16-64). No statistical difference was found between males and 
Table 1. Characteristics of Opisthorchis viverrini infections in participants in the starting year (2017)

\begin{tabular}{lrcc}
\hline Characteristics & No. samples & Infected (\%) & Average EPG (range) \\
\hline Gender & & & \\
Female & 2,428 & 13.7 & $49.0(25-683)$ \\
Male & 1,246 & 15.4 & $144.0(46-2,274)^{\mathrm{a}}$ \\
Age (yr) & & & \\
$20-30$ & 117 & $22.2^{\mathrm{a}}$ & $64.1(44.5-690)$ \\
$31-40$ & 1,066 & 12.5 & $76.4(60-492)$ \\
$41-50$ & 1,285 & 19.3 & $105.7(25-2,274)^{\mathrm{a}}$ \\
$51-60$ & 632 & 11.7 & $46.1(30-218)$ \\
$>60$ & 574 & 7.6 & $56.4(47-122)$ \\
Occupation & & & \\
Agriculture & 2,644 & 13.7 & $62.1(25-844)$ \\
Fishermen & 315 & $25.7^{\mathrm{a}}$ & $169.4(49-2,274)^{\mathrm{a}}$ \\
Government & 174 & 19.5 & $87.7(64-741)$ \\
Others & 541 & 9.0 & $98.4(53-288)$ \\
Province & & & \\
Nong Khai & 585 & 11.6 & $82.2(25-473)$ \\
Bueng Kan & 486 & 15.2 & $61.4(53-640)$ \\
Sakon Nakhon & 912 & 16 & $125.5(68-2,274)^{\mathrm{a}}$ \\
Nong Bua Lam Phu & 530 & 13.4 & $57.5(47-532)$ \\
Udon Thani & 1,161 & 14.3 & $68.7(60-408)$ \\
Total & 3,674 & 14.3 & $83.7(25-2,274)$ \\
\hline
\end{tabular}

females. However, an average intensity in males (EPG 28.0, range 16-64) was slightly higher than in females (EPG 16.7, range 14-23) $(P=0.032)$. The highest reinfection in the second year was among fishermen (23.2\%, EPG 26.8, range 18-52). The incidence of reinfection was found statistically highest in Udon Thani Province. However, the highest intensity of reinfection was detected in Sakon Nakhon Province. The new incidence of $O$. viverrini infection in second year from uninfected citizens $(\mathrm{n}=250)$ was less than $0.5 \%$ (data not shown).

In the third year, after praziquantel treatment and providing health education, prevalence of reinfection was 6.3\% (EPG 20.4, range 16-46). It was still high but not statistically significant among participants by gender, age, occupation, and location. Average intensity of the reinfected patients was more prominent in males (EPG 23.0, range 17-46), in 40-50 years old (EPG 21.4, range 17-46), fishermen (EPG 18.5, range 16-25), and in those who live in Udon Thani province (EPG 21.0, range 1846). The spatial distribution of human O. viverrini infection in the third year (2019) is shown in Fig. 1.

astatistically significant at $P<0.05$.

Percent of prevalence
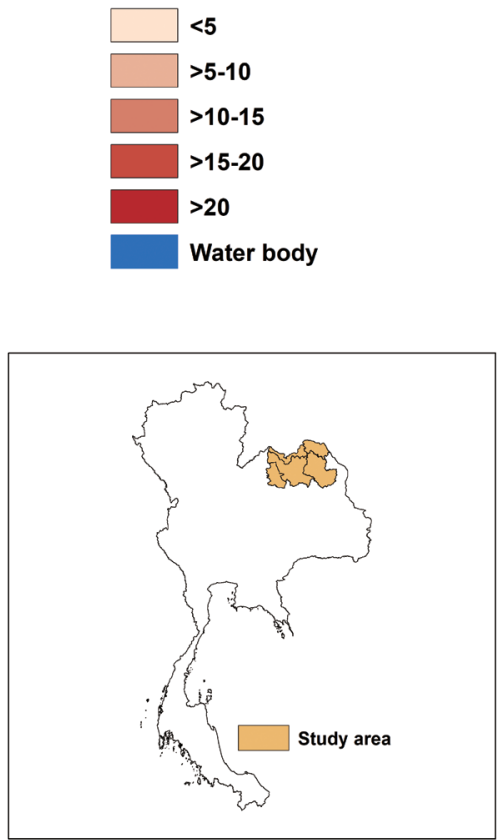
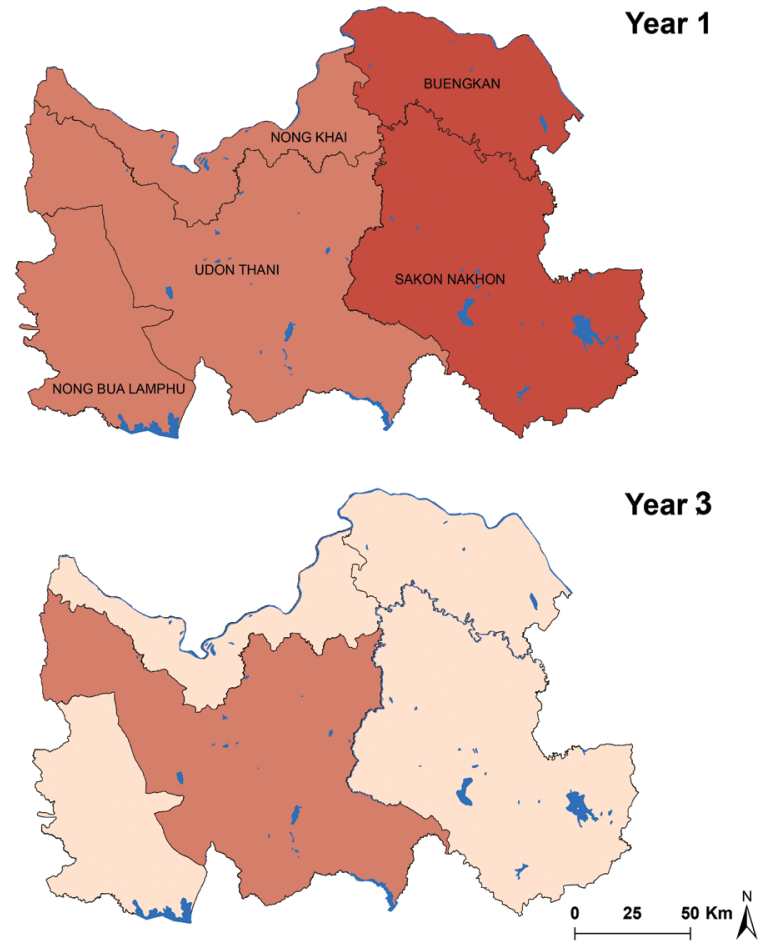

Fig. 1. Prevalence and percent of reinfection of Opisthorchis viverrini infections in an endemic area of Thailand. The distribution of infections is shown for each province. For reinfection, the samples were collected in the second and third year from participants infected in the first year. 
Table 2. Characteristics of opisthorchiasis in the reinfection populations in the proceeding years (2018 and 2019)

\begin{tabular}{|c|c|c|c|c|c|c|c|}
\hline \multirow[b]{2}{*}{ Characteristics } & \multirow{2}{*}{$\begin{array}{l}\text { No. of infections } \\
\text { in the first year }\end{array}$} & \multicolumn{3}{|c|}{ Reinfection in the second year } & \multicolumn{3}{|c|}{ Reinfection in the third year } \\
\hline & & No. of samples & $\begin{array}{l}\text { Percent of } \\
\text { reinfected (\%) }\end{array}$ & $\begin{array}{c}\text { Average EPG } \\
\text { (range) }\end{array}$ & No. of samples & $\begin{array}{l}\text { Percent of } \\
\text { reinfected (\%) }\end{array}$ & $\begin{array}{c}\text { Average EPG } \\
\text { (range) }\end{array}$ \\
\hline \multicolumn{8}{|l|}{ Gender } \\
\hline Female & 334 & 274 & 13.8 & $16.7(14-23)$ & 198 & 4.5 & $18.0(16-32)$ \\
\hline Male & 192 & 145 & 18.6 & $28.0(16-64)^{\mathrm{a}}$ & 116 & 6.9 & $23.0(17-46)$ \\
\hline \multicolumn{8}{|l|}{ Age (yr) } \\
\hline $20-30$ & 26 & 19 & 10.5 & $20.0(19-21)$ & 14 & 7.1 & $18.0(18-18)$ \\
\hline $31-40$ & 134 & 114 & 8.7 & $16.2(17-36)$ & 92 & 4.3 & $18.5(16-34)$ \\
\hline $41-50$ & 248 & 186 & $22.5^{\mathrm{a}}$ & $24.2(18-64)$ & 139 & 7.9 & $21.4(17-46)$ \\
\hline $51-60$ & 74 & 65 & 13.8 & $15.0(16-54)$ & 48 & 2.0 & $19.0(19-19)$ \\
\hline$>60$ & 44 & 35 & 5.7 & $18.5(17-20)$ & 21 & 0 & $0.0(0-0)$ \\
\hline \multicolumn{8}{|l|}{ Occupation } \\
\hline Agriculture & 362 & 318 & 14.4 & $20.2(16-64)$ & 237 & 5.0 & $21.1(17-46)$ \\
\hline Fishermen & 81 & 56 & $23.2^{\mathrm{a}}$ & $26.8(18-52)$ & 47 & 8.5 & $18.5(16-25)$ \\
\hline Government & 34 & 29 & 19.5 & $18.2(17-24)$ & 20 & 5.0 & $18.0(18-18)$ \\
\hline Others & 49 & 16 & 6.2 & $22.0(22-22)$ & 10 & 0 & $0.0(0-0)$ \\
\hline \multicolumn{8}{|l|}{ Province } \\
\hline Nong Khai & 68 & 57 & 5.2 & $18.0(16-19)$ & 41 & 2.4 & $20.0(20-20)$ \\
\hline Bueng Kan & 74 & 68 & 11.7 & $19.2(17-36)$ & 55 & 3.6 & $19.5(16-23)$ \\
\hline Sakon Nakhon & 146 & 120 & 15.8 & $28.4(18-64)^{\mathrm{a}}$ & 90 & 3.3 & $18.3(17-20)$ \\
\hline Nong Bua Lam Phu & 71 & 62 & 17.9 & $17.8(17-21)$ & 48 & 4.1 & $21.5(21-22)$ \\
\hline Udon Thani & 167 & 112 & $21.4^{\mathrm{a}}$ & $18.6(17-24)$ & 80 & 11.2 & $21.0(18-46)$ \\
\hline Total & 526 & 419 & 15.5 & $21.4(16-64)$ & 314 & 6.3 & $20.4(16-46)$ \\
\hline
\end{tabular}

astatistically significant at $P<0.05$.

Table 3. Prevalences and infection intensities of Opisthorchis viverrini in reservoir hosts and humans

\begin{tabular}{lccc}
\hline Host & $\begin{array}{c}\text { No. of samples } \\
\text { examined }\end{array}$ & $\begin{array}{c}\text { No. of infected } \\
(\%)\end{array}$ & $\begin{array}{c}\text { Mean EPG } \\
\text { (range) }\end{array}$ \\
\hline Dogs & 247 & $38(15.3)^{\mathrm{a}}$ & $35.8(26-142)$ \\
Cats & 61 & $7(11.4)$ & $54.4(32-168)^{\mathrm{a}}$ \\
Humans & 3,674 & $526(14.3)$ & $83.7(25-2,274)^{\mathrm{a}}$ \\
Total & 3,982 & $571(14.3)$ & $75.4(25-2,274)$ \\
\hline
\end{tabular}

a $P<0.05$.

\section{Health education to participants}

After health education in the first and second years of the survey, the mean scores of the participants of all issues was not significantly different when compared pre-and post-tests $(P>0.05)$. After intervention in the third year, the mean scores of the post-test (7.4 from 10.0) were statistically significant in terms of knowledge, attitude and preventative behavior compared to the pre-test (5.2 from 10.0) $(P<0.05)$.

\section{Prevalence and intensity of infection in reservoir hosts}

Prevalence in reservoir hosts is shown in Table 3. Liver fluke infections in dogs $(15.3 \%)$ were significantly higher than in cats $(11.4 \%) P<0.05)$. An average EGP in village dogs was
35.8 (26-142), in cats 54.4 (32-168) and in villagers 83.7 (25$2,274)$. Significant differences were found in dogs compared to humans and cats $(P<0.05)$.

The association of liver fluke infections in dogs and their owners was analyzed using Pearson's correlation for parametric data. In the first year, 33 households were home to 45 infected dogs and cats. No statistically significant correlation between simultaneous $O$. viverrini infection in these 33 households $(P>0.05, r=0.534)$ was found. Infection intensity of the owners (EPG 67.8, range 52-382) was positively associated with that of their dogs (35.8, range 26-142), but not statistically correlated $(P>0.05, r=0.672)$.

\section{Relative risk factors of $O$. viverrini reinfection}

To investigate the predictive risk of opisthorchiasis reinfection, we carried out a logistic regression analysis and calculated odds ratios (OR) on the 340 collected questionnaires (64.6\% of infected participants). Reinfection of $O$. viverrini was significantly related to gender, age, occupation, consumption of uncooked fish within a year of the study $(P \leq 0.05$; Table 4$)$. Most of participants were prominently liver fluke infected males. Their numbers were statistically different than for females 
Table 4. Relative risk factors for Opisthorchis viverrini reinfection in the second year

\begin{tabular}{|c|c|c|c|c|}
\hline Factors & $\begin{array}{l}\text { No. of questionnaires } \\
\qquad(n=340)\end{array}$ & Infected (\%) & $P$-value & Odds ratio $(95 \% \mathrm{Cl})$ \\
\hline \multicolumn{5}{|l|}{ Gender } \\
\hline Female & 242 & 14.8 & 0.017 & 1.0 \\
\hline Male & 108 & 22.2 & & $2.1(1.2-3.9)$ \\
\hline \multicolumn{5}{|l|}{ Age (yr) } \\
\hline$<40$ & 127 & 12.5 & 0.010 & 1.0 \\
\hline$>40$ & 213 & 20.6 & & 2.6(1.4-4.2) \\
\hline \multicolumn{5}{|l|}{ Education } \\
\hline Higher than Primary school & 73 & 15.0 & 0.271 & 1.0 \\
\hline Primary school and under & 267 & 18.3 & & $1.1(0.6-1.3)$ \\
\hline \multicolumn{5}{|l|}{ Occupation } \\
\hline Government and Others & 43 & 9.3 & 0.041 & 1.0 \\
\hline Fishermen and Farmer & 297 & 18.8 & & $2.4(0.9-11.6)$ \\
\hline \multicolumn{5}{|l|}{ Know use of praziquantel } \\
\hline Yes & 187 & 10.2 & 0.147 & 1.0 \\
\hline No & 153 & 16.4 & & $1.6(0.7-7.4)$ \\
\hline \multicolumn{5}{|c|}{ Eating uncooked fish in previous year } \\
\hline Never & 196 & 15.1 & 0.000 & 1.0 \\
\hline Often & 144 & 34.7 & & $3.5(1.4-16.6)$ \\
\hline
\end{tabular}

$(P=0.017, \mathrm{OR}=2.1)$. Those over 40 years old had an increased risk of liver fluke reinfection $(P=0.010, \mathrm{OR}=2.6)$. A higher risk was detected in fishermen and farmers compared to participants working in the government and other areas $(P=0.041$, $\mathrm{OR}=2.4$ ). Consumption of raw fish within the past year was a predictive risk of reinfection in the study areas $(P \leq 0.001$, $\mathrm{OR}=3.5$ ). Medical records revealed that many of those who were reinfected had previously been treated with praziquantel $(P=0.147, \mathrm{OR}=1.6)$. Additionally, other risk factors included education level $(P=0.271, \mathrm{OR}=1.1)$, alcohol consumption $(P=0.086, \mathrm{OR}=1.2)$, smoking $(P=0.162, \mathrm{OR}=1.4)$ and salary $(P=0.146, O R=1.3)$, but these factors were not statistically significant (data not shown).

\section{DISCUSSION}

We first investigated the profiles of the prospective cohort in this study, the 3-year management of $O$. viverrini infection and reinfection associated risk factors in the upper part of Northeastern Thailand. A previous epidemiological study in this endemic region revealed a high prevalence of $O$. viverrini in various habitats, especially in water basin areas such as those of Lawa Lake in Khon Kaen Province (70\%) [6], Huay Luang Reservoir in Udon Thani Province (31.5\%) [7], and the Me Kong Basin in Nakhon Panom Province (60.0\%) [14]. Changing patterns of the prevalence in $O$. viverrini infection in children and ado- lescents in Northeastern Thailand were reported [11]. Our findings also show the highest infection in the age 20-30 years old group and the average intensity significantly peaked among 4150 years old. This finding changed over the past 10 years in that people over 40 years old [14]. That change may involve the risks identified. A previous study conducted by a previous National Control Program identified 30 year olds and above as a high risk group. Additionally, people of this age group usually move away from their birthplace. The progressive risk of liver fluke infection may increase in the next generation.

Reinfection and chronic infection of liver fluke induces hepatobiliary diseases as well as cholangiocarcinoma [15-17]. In our previous report, the pathology of opisthorchiasis after praziquantel treatment was diminished, as has been demonstrated in hamsters $[18,19]$. The important finding in this study is that when we gave health education and administered a control program to the community, the percent of reinfection was persistent at $15.5 \%$ (65 infected) in the second year and 6.3\% (17 infected) in the third year. Effective treatment with praziquantel may have reinforced incorrect attitudes towards eating uncooked fish [20]. Additionally, adverse effects of the immune response have been demonstrated within the bile duct epithelial cells after treatment with praziquantel in the short term [21]. Participants reinfected with O. viverrini may have increased risk of cholangiocarcinoma development [14]. After health education in the first and second years of the study, the mean 
scores of the knowledge, attitude and practice of participants were not significantly different comparing the pre- and posttests $(P>0.05)$. However, with intervention in the third year, the mean scores of the post-test were significantly higher than the pre-test. This finding was related to the significantly diminished O. viverrini reinfections in patients. Changes in the health education program enhanced awareness, reducing the reinfection rate in the endemic areas. Reinfection by $O$. viverrini has been linked to the number of praziquantel treatments in the community [3]. However, our observations indicated that some people in this region can purchase praziquantel and take it directly without laboratory diagnosis $[5,7]$. Our study provides strong evidence of the reinfection profile in the community. In the second year of this study, the incidence of new $O$. viverrini infections among the uninfected citizens was less than $0.5 \%$. Whereas, the reinfection was $15.5 \%$. This decreased to $6.3 \%$ in the third year of the study. In those with first time liver fluke infections, with treatment and health education, the participants came to understand the severity of opisthorchiasis as well as of cholangiocarcinoma [22]. However, their lifestyles led them to become reinfected due to their cultural behaviors [23]. The highest level of reinfection in the second year was among fisherman. In the first year, the prevalence and intensity were highest in Sakon Nakhon and Bueng Kan Provinces. However, reinfection was commonly found in Udon Thani Province in the second and third years. This high reinfection rate may involve a high contamination of metacercaria in cyprinid fish in this province as previously reported [9]. Behavioral risks were commonly found in these areas and more intensive prevention and control programs are needed. Additionally, some of participants exited the study by changing their workplace. These groups did not receive health education as infected participants, and may have become reinfected. In this case, the severity of opisthorchiasis may have been underestimated [22].

Praziquantel was a drug of choice for treatment against parasitic trematodes. The cure rate with $40 \mathrm{mg} / \mathrm{kg}$ body weight after 30 days was $100 \%$ [24]. Whereas, in the heavy infections with EPG 10,800-139,000, the cure rate using $50 \mathrm{mg} / \mathrm{kg}$ body weight was $97.0 \%$ [25]. In this study, no one was O. viverrini-egg positive after one month of treatment. Reinfection in participants in this study occurred during the year after previous treatment.

Our previous study suggested that although liver fluke was treated in humans, it remained in the reservoir hosts such as dogs and cats, which are raised in the endemic areas [5]. For the purpose of environmental management, dogs and cats were diagnozed and treated for $O$. viverrini. Our findings showed liver fluke infections in humans and their dogs or cats were common near bodies of water. Additionally, the association of $O$. viverrini infection in dogs and villagers was found when the owners fed some pieces or uneaten parts of raw fish to domestic animals [26]. The association of the diet of a family and sharing food with dogs is an embedded cultural practice [27]. The infection in dogs and cats was $1.6 \%$ and $1.3 \%$, respectively. With respect to animal defecation, cats usually drop their stools indiscriminately, contaminating the areas around bodies of water with liver fluke eggs, continuing the life cycle of these parasites $[5,7]$. Therefore, a strategy of prevention and control to eliminate contamination from only human subjects is inadequate.

The recognized risk factors of liver fluke infection have been identified, including the practice of consuming raw fish in many kinds of traditional dishes [28]. In our study, 50 participants who consumed raw fish within the previous year were positively diagnosed with liver fluke eggs in the second year. Reinfection of $O$. viverrini was significantly related to the male gender, those aged over 40 years old, those who were fishermen, and consumed uncooked fish within the previous year of the study $(P \leq 0.05)$. Studies of the infection profile showed that about $86 \%$ of those treated for $O$. viverrini infection were reinfected within one year [29]. The highest reinfection in the second year was among fishermen and those living around water bodies. Increased risk was associated with eating raw fish. Those living near the Nong Han Wetlands in Udon Thani and the Nam Oon Reservoir had the highest reinfection rate and intensity. The second intermediate hosts, fresh water fish containing the metacercariae, were widely distributed, increasing the range of infection [30].

A negative correlation between educational level and alcohol consumption was observed with $O$. viverrini reinfection. One speculation is that the more highly educated people tend to have greater social mobility, but still had strong cultural pressure to consume traditional dishes serving unsafely prepared fish [31]. Consumption of raw fish within the past year was a predictive risk of reinfection in the study areas. In our observations, use of praziquantel is well-known $(P=0.147$; $\mathrm{OR}=1.6$ ), but this is not associated with liver fluke reinfection. People enjoy the flavor of uncooked fish and believe that fluke infestations can be eliminated by anthelmintic drugs, thereby removing the risk of cancer [20], leading to continuation of their behavior and reinfection. 
The activities of this study were a part of a National Control Program of the Ministry of Public Health, Thailand. The data derived from this study were reported to a national database and the patients were referred for further procedures. In conclusion, the prevalence of $O$. viverrini in the first year was $14.3 \%$ among 526 participants. They were treated using praziquantel and provided with health education and intervention. Significant differences in the results of pre- and post-tests were found in the third year of the study that show reduction of reinfection during that period. The reinfection rate was prominently in the age range of 41-50-year olds and those working as fishermen. Human reinfection rates were $15.5 \%$ in the second year and the reinfection level in the third year of the study was diminished to $6.3 \%$. However, the percentage of infection was still high but was not statistically related to any particular variables. Additionally, other gastrointestinal parasitic infections were reported, and a low rate of repeat infection was found. This study indicates that reinfection in the community and the epidemiology of $O$. viverrini is demonstrated in reservoir hosts. Our findings provide information for future effective and sustainable strategies to promote public health education campaigns. Active and sustained surveillance needs to be continuously performed for at least 3 years to decrease the risk of reinfection.

\section{ACKNOWLEDGEMENTS}

This study was supported by the Research and Development Institute Udon Thani Rajabhat University. We also thank Professor Dr. Jeffrey Nash from the Faculty of the Graduate School, Udon Thani Rajabhat University, for English editing of the manuscript.

\section{CONFLICT OF INTEREST}

We declare that there are no conflicts of interest in this work.

\section{REFERENCES}

1. Sripa B, Bethony JM, Sithithaworn P, Kaewkes S, Mairiang E, Loukas A, Mulvenna J, Laha T, Hotez PJ, Brindley PJ. Opisthorchiasis and Opisthorchis-associated cholangiocarcinoma in Thailand and Laos. Acta Trop 2011; 120 (suppl): 158-168.

2. Pinlaor S, Ma N, Hiraku Y, Yongvanit P, Semba R, Oikawa S, Murata M, Sripa B, Sithithaworn P, Kawanishi S. Repeated infection with Opisthorchis viverrini induces accumulation of 8-nitroguanine and 8-oxo-7,8-dihydro-2'-deoxyguanine in the bile duct of hamsters via inducible nitric oxide synthase. Carcinogenesis 2004; 25: 15351542.

3. Thinkhamrop K, Khuntikeo N, Sithithaworn P, Thinkhamrop W, Wangdi K, Kelly MJ, Suwannatrai AT, Gray DJ. Repeated praziquantel treatment and Opisthorchis viverrini infection: A population-based cross-sectional study in northeast thailand. Infect Dis Poverty 2019; 8: 18 .

4. Kamsa-Ard S, Luvira V, Pugkhem A, Luvira V, Thinkhamrop B, Suwanrungruang K, Bhudhisawasdi V. Association between praziquantel treatment and cholangiocarcinoma: A hospital-based matched case-control study. BMC Cancer 2015; 15: 776.

5. Prakobwong S, Suwannatrai A, Sancomerang A, Chaipibool S, Siriwechtumrong N. A large scale study of the epidemiology and risk factors for the carcinogenic liver fluke Opisthorchis viverrini in Udon Thani Province, Thailand. Asian Pac J Cancer Prev 2017; 18: 2853-2860.

6. Sripa B, Tangkawattana S, Laha T, Kaewkes S, Mallory FF, Smith JF, Wilcox BA. Toward integrated opisthorchiasis control in northeast thailand: The lawa project. Acta Trop 2015; 141: 361-367.

7. Prakobwong SGW, Chaipibool S, Nimala B, Sangthopho J, Sirivetthumrong N, Ribas A. Epidemiology of Opisthorchis viverrini in an endemic area of thailand, an integrative approach. Helmithologia 2017; 54: 298-306.

8. Nithiuthai S, Suwansaksri J, Wiwanitkit V, Chaengphukeaw P. A survey of metacercariae in cyprinoid fish in Nakhon rRatchasima, northeast thailand. Southeast Asian J Trop Med Public Health 2002; 33 (suppl): 103-105.

9. Pinlaor S, Onsurathum S, Boonmars T, Pinlaor P, Hongsrichan N, Chaidee A, Haonon O, Limviroj W, Tesana S, Kaewkes S, Sithithaworn P. Distribution and abundance of Opisthorchis viverrini metacercariae in cyprinid fish in northeastern Thailand. Korean J Parasitol 2013; 51: 703-710.

10. Grundy-Warr C, Andrews RH, Sithithaworn P, Petney TN, Sripa B, Laithavewat L, Ziegler AD. Raw attitudes, wetland cultures, life-cycles: Socio-cultural dynamics relating to Opisthorchis viverrini in the mekong basin. Parasitol Int 2012; 61: 65-70.

11. Khuntikeo N, Sithithaworn P, Loilom W, Namwat N, Yongvanit P, Thinkhamrop B, Kiatsopit N, Andrews RH, Petney TN. Changing patterns of prevalence in Opisthorchis viverrini sensu lato infection in children and adolescents in northeast thailand. Acta Trop 2016; 164: 469-472.

12. Saengsawang P, Promthet S, Bradshaw P. Reinfection by Opisthorchis viverrini after treatment with praziquantel. Asian Pac J Cancer Prev 2016; 17: 857-862.

13. Suwannatrai A, Saichua P, Haswell M. Epidemiology of Opisthorchis viverrini infection. Adv Parasitol 2018; 101: 41-67.

14. Sithithaworn P, Andrews RH, Nguyen VD, Wongsaroj T, Sinuon M, Odermatt P, Nawa Y, Liang S, Brindley PJ, Sripa B. The current status of opisthorchiasis and clonorchiasis in the Mekong Basin. Parasitol Int 2012; 61: 10-16.

15. Flavell DJ, Flavell SU. Opisthorchis viverrini: Pathogenesis of infection in immunodeprived hamsters. Parasite Immunol 1986; 8: 455- 
466.

16. Prakobwong S, Pinlaor S, Yongvanit P, Sithithaworn P, Pairojkul C, Hiraku Y. Time profiles of the expression of metalloproteinases, tissue inhibitors of metalloproteases, cytokines and collagens in hamsters infected with Opisthorchis viverrini with special reference to peribiliary fibrosis and liver injury. Int J Parasitol 2009; 39: 825-835.

17. Prakobwong S, Yongvanit P, Hiraku Y, Pairojkul C, Sithithaworn P, Pinlaor P, Pinlaor S. Involvement of mmp-9 in peribiliary fibrosis and cholangiocarcinogenesis via rac1-dependent DNA damage in a hamster model. Int J Cancer 2010; 127: 2576-2587.

18. Pinlaor S, Prakobwong S, Boonmars T, Wongkham C, Pinlaor P, Hiraku Y. Effect of praziquantel treatment on the expression of matrix metalloproteinases in relation to tissue resorption during fibrosis in hamsters with acute and chronic Opisthorchis viverrini infection. Acta Trop 2009; 111: 181-191.

19. Mairiang E, Haswell-Elkins MR, Mairiang P, Sithithaworn P, Elkins DB. Reversal of biliary tract abnormalities associated with Opisthorchis viverrini infection following praziquantel treatment. Trans $\mathrm{R}$ Soc Trop Med Hyg 1993; 87: 194-197.

20. Wongba N, Thaewnongiew K, Phathee K, Laithavewat L, Duangsong R, Promthet S, Tangsawad S. Liver fluke prevention and control in the northeast of Thailand through action research. Asian Pac J Cancer Prev 2011; 12: 1367-1370.

21. Pinlaor S, Prakobwong S, Hiraku Y, Kaewsamut B, Dechakhamphu S, Boonmars T, Sithithaworn P, Pinlaor P, Ma N, Yongvanit $\mathrm{P}$, Kawanishi S. Oxidative and nitrative stress in Opisthorchis viverrini -infected hamsters: An indirect effect after praziquantel treatment. Am J Trop Med Hyg 2008; 78: 564-573.

22. Kaewpitoon SJ, Kaewpitoon N, Rujirakul R, Ueng-Arporn N, Matrakool L, Tongtawee T. The carcinogenic liver fluke Opisthorchis viverrini among rural community people in northeast Thailand: A cross- sectional descriptive study using multistage sampling technique. Asian Pac J Cancer Prev 2015; 16: 7803-7807.

23. Painsing S, Sripong A, Vensontia O, Pengsaa P, Kompor P, Kootanavanichapong N, Kaewpitoon SJ, Kaewpitoon N. Health behavior regarding liver flukes among rural people in Nakhon Ratchasima, Thailand. Asian Pac J Cancer Prev 2016; 17: 21112114.

24. Pungpak S, Radomyos P, Radomyos BE, Schelp FP, Jongsuksuntigul P, Bunnag D. Treatment of Opisthorchis viverrini and intestinal fluke infections with praziquantel. Southeast Asian J Trop Med Public Health 1998; 29: 246-249.

25. Pungpak S, Bunnag D, Harinasuta T. Studies on the chemotherapy of human opisthorchiasis: Effective dose of praziquantel in heavy infection. Southeast Asian J Trop Med Public Health 1985; 16: 248-252.

26. Aunpromma S, Tangkawattana P, Papirom P, Kanjampa P, Tesana S, Sripa B, Tangkawattana S. High prevalence of Opisthorchis viverrini infection in reservoir hosts in four districts of Khon Kaen Province, an opisthorchiasis endemic area of Thailand. Parasitol Int 2012; 61: 60-64.

27. Chudthaisong N, Promthet S, Bradshaw P. Risk factors for opisthorchis viverrini infection in Nong Khai Province, thailand. Asian Pac J Cancer Prev 2015; 16: 4593-4596.

28. Sripa B, Kaewkes S, Sithithaworn P, Mairiang E, Laha T, Smout M, Pairojkul C, Bhudhisawasdi V, Tesana S, Thinkamrop B, Bethony JM, Loukas A, Brindley PJ. Liver fluke induces cholangiocarcinoma. PLoS Med 2007; 4: e201.

29. Upatham ES, Viyanant V, Brockelman WY, Kurathong S, Lee P, Kraengraeng R. Rate of re-infection by Opisthorchis viverrini in an endemic northeast Thai community after chemotherapy. Int J Parasitol 1988; 18: 643-649.

30. Sous SM. Iinfluence of environmental factors on the infection rates of fishes and shellfishes by trematodes of the Opisthorchidae Family in the Krotovaya Lyaga Lake. Med Parazitol (Mosk) 2006: 41-46 (in Russian).

31. Chaiputcha K, Promthet S, Bradshaw P. Prevalence and risk factors for infection by Opisthorchis viverrini in an urban area of Mahasarakham Province, northeast Thailand. Asian Pac J Cancer Prev 2015; 16: 4173-4176. 
